\title{
Bioterror: Wie groß ist die Gefahr wirklich?
}

\author{
Auch wenn es bisher nicht zu großen, flächendeckenden Anschlägen mit biologischen Waffen \\ gekommen ist und etwa die Attacken mit Anthrax-verseuchten Briefen in den USA schon \\ einige Jahre zurückliegen - das Risiko Bioterror ist nicht aus der Welt. Von welchen Erregern \\ gehen die größten Gefahren aus? Und: Sind wir darauf vorbereitet?
}

\begin{abstract}
Substanzen für einen bioterroristischen Anschlag sollten sich durch Stabilität, hohe Letalität und möglichst fehlendes Gegenmittel auszeichnen, erklärte Prof. Dr. Rüdiger Braun, Laborarzt aus Ludwigsburg.
\end{abstract}

\section{Botinulinumtoxin im \\ Trinkwasser?}

Toxine eignen sich in dieser Hinsicht als Waffe, allerdings vermehren sie sich nicht wie Viren oder Bakterien und werden nicht von Mensch zu Mensch übertragen. Wäre es beispielsweise möglich, Trinkwasser mit Botulinumtoxin, einem der stärksten bekannten Toxine, zu vergiften? Schließlich genügt $1 \mathrm{mg}$ Botulinustoxin A, um 10.000 Menschen zu töten. In Deutschland wird aber das Trinkwasser über Fällung gereinigt, beruhigte Braun. Dadurch werden alle im Wasser befindlichen Proteine ausgefällt, hinzu kommt eine Transportchlorierung als zweite Sicherheit.

\section{Mögliche Bedrohung durch Pockenviren}

Für Viren als Biowaffen spricht die Mög lichkeit der Vermehrung. Allerdings sind sie nicht umweltstabil, und gegen viele Viren ist eine Impfung möglich.

Pockenviren sind fast komplett aus dem Fokus verschwunden, was aber nicht bedeutet, dass es sie nicht mehr gibt. Seit die Pflichtimpfung gegen Pocken 1980 abgeschafft wurde, fehlt einem großen Teil der Bevölkerung der entsprechende Schutz. Pockenviren gelten als relativ umweltstabil mit einer Überlebens-

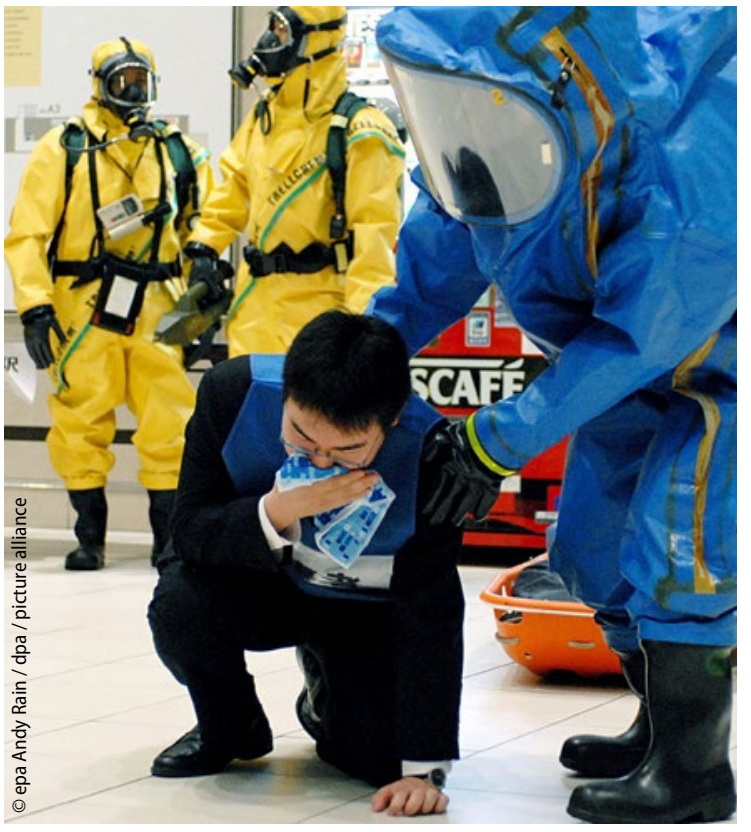

Anti-Bioterror-Übung in Tokio.

dauer von einem Tag bei Raumtemperatur und bis zu 14 Tagen im Winter. Die Letalität bei Infektion beträgt zwischen 20 und 30\%. Da in Labors in den USA und Russland immer noch Bestände von Pockenviren vorhanden sind, stellen diese Erreger tatsächlich eine Bedrohung dar, so Braun. Über die Wirksamkeit von antiviralen Mitteln (z. B. Cidofovir) lässt sich nur spekulieren.

\section{Pest und Milzbrand}

Bakterien können von Mensch zu Mensch übertragen werden und lassen sich leicht vermehren. Sie sind aber oft nur wenig stabil und kaum letal. Als Beispiele nannte Braun Milzbrand und Tularämie. Die Übertragung von Anthraxsporen erfolgt über direkten Kontakt, eine Nosokomialinfektion ist nicht mög- lich. Die Infektion verläuft schwer, vor allem, wenn sie zu spät bemerkt wird. Anthrax aber lässt sich antibiotisch mit Ciprofloxacin (2 x 400 mg i.v.) behandeln.

Ein weiterer potenzieller Kandidat für eine Biowaffe wäre Yersinia pestis. Immer noch gibt es Pestherde in den USA, insbesondere in Kalifornien unter den Präriehunden. Auch auf Madagaskar kommt es regelmäßig zu Pestinfektionen, sodass es nicht schwierig wäre, an den Erreger heranzukommen.

\section{Gefahr durch rekombinante Krankheitserreger}

Einen neueren Ansatz für potenzielle Biowaffen stellen genetisch veränderte Organismen dar. Im Reagenzglas kann man sich einen Organismus zurechtschneiden mit unter Umständen hoher Letalität und ggf. rascher Verbreitung, so Braun. Demgegenüber stehen allerdings die hohen technischen Hürden und das notwendige Expertenwissen. Eckard Wimmer gelang 1999 die komplette In-vitro-Synthese eines Enterovirus. Auch das Influenzavirus von 1918 wurde nachgebaut.

Generell gilt, dass sich Biowaffen nicht leicht herstellen und verbreiten lassen. Daher sieht Braun derzeit nur eine geringe Bedrohung. Die Möglichkeiten der Biotechnologie könnten aber in $\mathrm{Zu}$ kunft bei missbräuchlicher Anwendung ein erhebliches Gefahrenpotenzial bilden.

Martin Bischoff

- 52. Ärztekongress, Stuttgart, 28. Januar 2017 